\section{$\underset{\substack{\text { hommes } \\ \text { \& migrations }}}{ }$}

\section{Hommes \& migrations}

Revue française de référence sur les dynamiques

migratoires

$1296 \mid 2012$

Le Mexique dans les migrations internationales

\title{
Migration et tradition
}

La fête patronale entre changement et continuité

\section{Patricia Arias}

Traducteur : Jean Hennequin

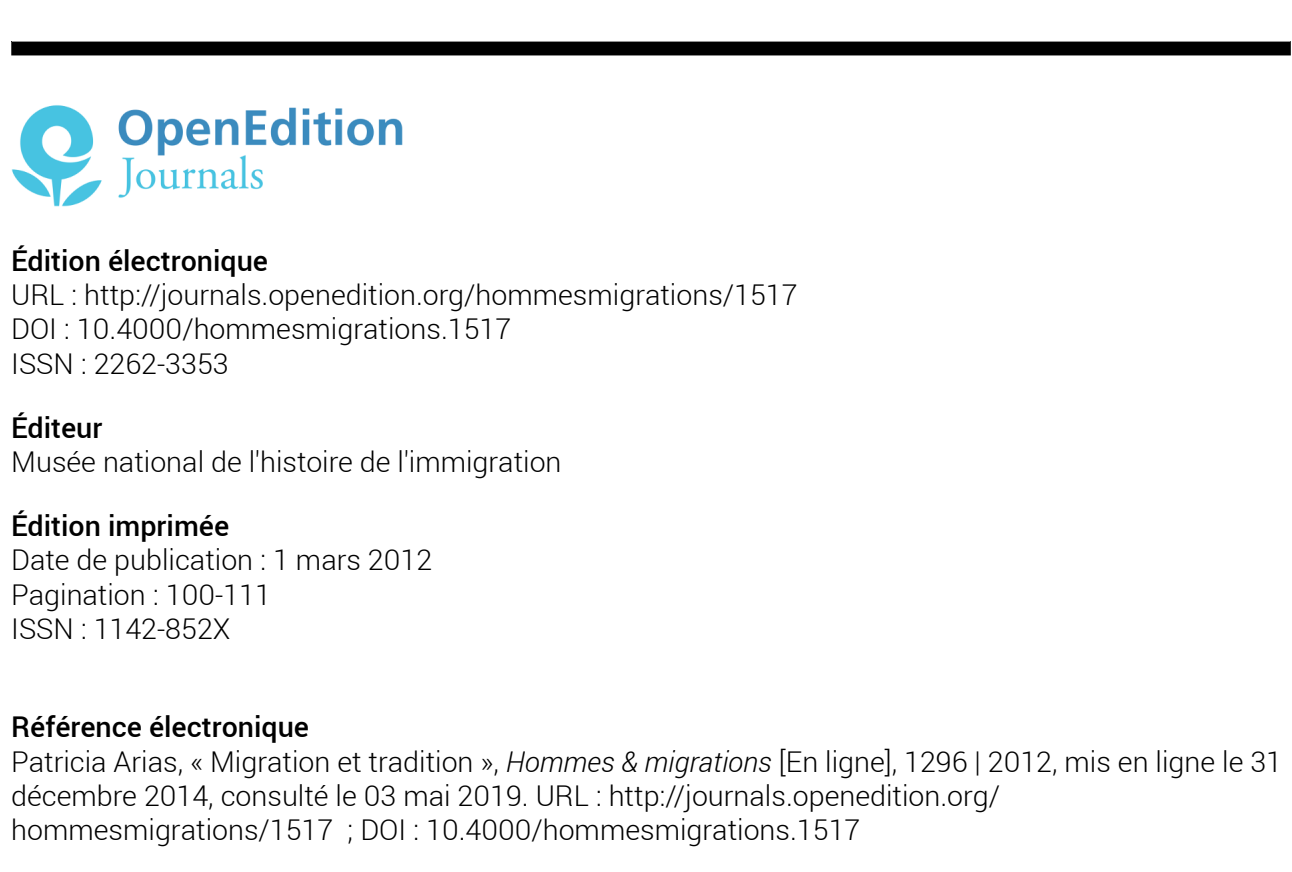

Tous droits réservés 


\section{Migration et tradition La fête patronale entre changement et continuité}

Par Patricia Arias, chercheuse en anthropologie sociale et géographie, université de Guadalajara

Au Mexique, dans les communautés rurales, les célébrations des fêtes patronales sont le point d'orgue du culte rendu aux saints. Organisées à l'échelle des villages dont elles mobilisent

les populations, elles permettent de célébrer la permanence

d'une identité commune. La participation de chacun est requise en fonction des responsabilités qu'il occupe dans la communauté. Non contents de faire des fêtes patronales l'occasion d'un retour, les Mexicains imposent aux migrants d'y participer financièrement.

Une exigence qui ne va pas sans heurts lorsque se distendent les liens avec le village d'origine. 
Les recherches récentes font état non seulement de la persistance des fêtes patronales, mais aussi d'un important changement qui les concerne : plus les communautés rurales sont pauvres, plus elles sont touchées par les processus de migration, aussi bien nationale qu'internationale, et plus leurs fêtes patronales sont coûteuses et spectaculaires ${ }^{(1)}$. Les ethnographies ont mis en évidence un faste que les fêtes ne présentaient pas à un tel point auparavant : les communautés font venir à grands frais des artistes, organisent des spectacles, des concours, à la fois nouveaux et différents de ceux qui étaient traditionnellement associés à la fête religieuse. Dans tous les cas, on cherche à ce que les migrants participent aux festivités et en supportent les frais.

Dans l'Amérique latine coloniale, les villages - qu'ils aient existés auparavant ou qu'ils soient de création récente - se sont vus attribuer un saint patron ou une sainte patronne tirés du vaste martyrologe catholique. Ils se sont ainsi entourés d'une image de dévotion et ont été dotés d'un temps réservé aux célébrations festives, reconnu aussi bien à l'intérieur qu'à l'extérieur des communautés : la fête patronale, autour de laquelle est réorganisé le calendrier civil et rituel de chacune d'entre elles. La fête patronale a permis aux communautés de conserver l'“ambiance de réjouissance propre aux anciennes fêtes" et a donné naissance à une nouvelle "hiérarchie sociale et politique [...] reposant sur la distribution des responsabilités religieuses catholiques ${ }^{(2) ", ~ h i e ́ r a r c h i e ~ q u i ~}$ en vint à se confondre avec l'organisation sociale méso-américaine ${ }^{(3)}$. Le système de distribution des responsabilités au sein de la communauté, exercé et hiérarchisé sous forme de mayordomías ${ }^{(4)}$, a été l'institution chargée d'organiser et de financer la fête patronale dans les communautés indiennes et rurales du Mexique ${ }^{(5)}$.

La fête patronale a subsisté parce qu'elle constitue un mécanisme souple qui a su intégrer des intérêts et des sens multiples, variables à travers le temps, l'espace et les changements qu'ont connus les communautés. De nos jours, les migrants nationaux et internationaux constituent une importante source de revenus - pour ne pas dire la principale - qui permet de financer les fêtes et d'assumer les responsabilités qui y sont liées au sein des communautés d'origine ${ }^{(6)}$.

\section{La fin de la circularité migratoire}

Les années quatre-vingt-dix ont été marquées par la convergence de trois processus qui ont entraîné des conséquences majeures pour les familles et les communautés rurales. En premier lieu, l'intensification et la généralisation de la migration, avec l'élargissement de l'espace géographique de la migration vers les États-Unis, ainsi que vers les villes, les centres touristiques et la frontière Nord du Mexique ${ }^{(7)}$. La migration est venue se grossir des contingents envoyés par les États fédérés possédant les plus 
importantes populations indigènes : Campeche, Chiapas, Guerrero, Oaxaca, Puebla, Tlaxcala, Veracruz, Yucatán ${ }^{(8)}$.

En second lieu, la "désagrarisation" des économies paysannes et le dépeuplement du monde rural. Les communautés ont perdu leurs activités économiques traditionnelles, sans que d'autres soient apparues pour les remplacer. D'où un processus de dépeuplement. Les jeunes des deux sexes s'en vont de leurs communautés où seules demeurent des personnes âgées et des familles dans des situations fort précaires.

En troisième lieu, la perte de pouvoir des communautés, liée à la fin de la distribution des terres en vertu de l'application de la loi agraire de 1992. La délivrance de titres individuels de propriété des ejidos ${ }^{(9)}$ impliqua la remise de la propriété aux personnes les plus âgées, qui en étaient les titulaires originaux. Dans ces conditions, les jeunes furent exclus de la possibilité d'accéder à des parcelles et terrains par héritage ou par dotation. Les communautés et les familles perdirent donc l'une de leurs principales sources de pouvoir et de contrôle sur les habitants, à savoir la dotation des terres ${ }^{(10)}$. En tout état de cause, la migration généralisée a donné naissance à des scénarios différents dans les anciennes et les nouvelles régions migratoires. Les migrants de la région historique, qui allaient et venaient depuis des décennies, furent les principaux bénéficiaires de l'amnistie de $1986^{(11)}$, grâce à laquelle ils légalisèrent leur séjour ${ }^{(12)}$. Les migrants désormais en situation légale prirent des engagements à long terme aux États-Unis : achat de maisons et de commerces, séjour permanent des enfants et des petits-enfants. Si ces migrants peuvent désormais revenir sans entrave au Mexique, ils ne le font plus avec la même périodicité ni la même fréquence qu'autrefois. Dans les États du centre-sud du Mexique, où la migration est montée en flèche dans les années quatre-vingt-dix, c'est la migration des sans-papiers qui prédomine et le scénario est contradictoire. Les communautés et les familles attendent encore le retour des migrants et les investissements de "migradollars ${ }^{(13) " ~ d a n s ~ l a ~ c o n s t r u c t i o n ~ d e ~ m a i s o n s ~}$ se maintiennent. D'autres auteurs reconnaissent que les migrants, pour la plupart paysans sans terre, ne retournent dans leur village qu'à l'occasion des fêtes patronales ou d'une crise familiale ${ }^{(14)}$, ou bien y retournent pour y rester quelques mois seulement ${ }^{(15)}$. Une consultation réalisée auprès d'une communauté zapotèque de l'État d'Oaxaca a révélé que plus d'un tiers des migrants (36,5\%) vivaient en dehors de la communauté depuis dix à dix-neuf ans ${ }^{(16)}$. Peu à peu, les jeunes femmes ont elles aussi commencé à partir, seules ou en couple ${ }^{(17)}$. La militarisation de la frontière Nord a contribué à faire disparaître la circularité qui, durant plus d'un siècle, avait constitué l'une des caractéristiques de la migration mexicaine ${ }^{(18)}$.

Ainsi, alors que la migration était naguère liée à des raisons professionnelles, concernait essentiellement les jeunes adultes masculins et était un phénomène 
circulaire s'achevant par le retour, elle est devenue un processus familial à long terme, le retour est devenu incertain, indéfini, voire n'est plus envisagé(19). La migration généralisée et irréversible est venue modifier les scénarios traditionnels, changement qui se manifeste dans les fêtes patronales. Les ethnographies récentes constatent les difficultés qu'implique la gestion de la migration et font état des stratégies mises en cuvre par les communautés afin de maintenir vivante la fête patronale.

\section{Les stratégies visant à assurer le maintien de la fête}

Dans la région historique de la migration, caractérisée par un catholicisme fortement enraciné, l'Église a joué un rôle capital dans les stratégies unissant les migrants à la fête patronale. La première de ces stratégies a consisté à encourager la célébration d'une "fête de l'émigrant", accompagnée d'une liturgie particulière, La "fête de l'émigrant" s'est popularisée dans les villages de l'ouest du Mexique. Le scénario du non-retour a donné lieu à un changement sémantique : on ne parle plus désormais de la fête du migrant, mais de celle des "enfants absents". Le but poursuivi par ce changement d'étiquette est de faire en sorte que les migrants ne se sentent pas comme des émigrés, mais qu'ils continuent à être les enfants du village et de la paroisse. La notion d'absence suggère un état transitoire, laissant ouverte la possibilité du retour, et met l'accent sur ceux qui attendent.

Une deuxième stratégie a consisté pour les prêtres à se rendre aux États-Unis auprès de leurs paroissiens absents, afin de les inviter à rentrer au pays et à participer à la fête. Là-bas, les prêtres célébraient "des baptêmes, des mariages et des confessions, mais [maintiennent] avant tout le contact direct avec leurs paroissiens ${ }^{(20) "}$.

Une troisième stratégie, plus récente, consiste à transférer les images saintes, ainsi que les célébrations elles-mêmes, vers les lieux de destination ${ }^{(21)}$. Ceci ne se produisait pas auparavant, étant donné que les migrants revenaient tous les ans dans leurs communautés, pour ensuite y rester définitivement : dans ces conditions, à quoi bon transférer les fêtes aux États-Unis? Maintenant les choses ont changé. Dans certaines paroisses de Chicago, région où abondent les migrants au statut légal, se sont popularisées les images de la Vierge de Guadalupe, du Saint Enfant d'Atocha, du Père Pro, de Saint Juan Diego ${ }^{(22)}$. Des répliques des images saintes ont pris le chemin des nouvelles destinations migratoires : New York, destination privilégiée des migrants de l'État de Puebla, ainsi que certaines localités rurales de la Floride ${ }^{(23)}$. On a beau affirmer que la fête "continue à être au Mexique", dans les faits on la célèbre de part et d'autre de la frontière ${ }^{(24)}$. 
Les autorités civiles ont, elles aussi, conçu des stratégies destinées à adapter la fête aux circonstances issues du nouveau modèle migratoire. Le pari à relever consistait à faire en sorte que les migrants, nationaux et internationaux, reviennent au pays, en mettant en exergue l'ambiance festive, ainsi que l'aspect touristique de la fête patronale. Les autorités et les chefs d'entreprise lancent des campagnes publicitaires susceptibles d'intéresser et d'attirer les petits-enfants et arrière-petits-enfants des migrants qui ne sont plus liés au village par des engagements aussi forts, mais qui peuvent aller s'y amuser en compagnie de leurs amis. Ce type de promotion n'est pas toujours du goût des autorités ecclésiastiques, des personnes âgées et des croyants qui s'offusquent du sens non religieux attribué à la fête... mais il n'y a pas grand-chose à y faire, d'autant plus que leurs récriminations n'ont guère d'écho: pour le plus grand nombre, c'est la manière de maintenir vivante la fête, et bien souvent aussi la seule occasion de l'année où il y a un afflux de visites, de revenus, de dépenses, de consommation.

La situation est différente dans les communautés possédant une forte tradition indigène, dans lesquelles l'influence de l'Église catholique est moindre et où la communauté a plus de poids. La migration généralisée et prolongée a donné lieu à diverses stratégies destinées à assurer la permanence, aussi bien du système de distribution des responsabilités religieuses au sein de la communauté, que des fêtes religieuses elles-mêmes. Si certains se font un véritable plaisir de revenir au village pour y assumer leur responsabilité vis-à-vis de l'église ${ }^{(25)}$, ce n'est pas le cas de la majorité. En réalité, les frais et les dettes qu'entraîne ce genre de responsabilité peuvent constituer un motif de migration ${ }^{(26)}$. Dans l'État d'Oaxaca, certaines communautés obligent leurs membres à revenir en personne au village lorsqu'ils sont désignés pour assumer de telles fonctions; d'autres ne font plus aucun cas de ceux qui ont quitté la communauté(27). Quoi qu'il en soit, il s'est ouvert un espace de tension et de redéfinition des mesures à adopter afin d'assurer le respect des engagements communautaires.

\section{Le prix des responsabilités traditionnelles}

Une solution pratique a consisté à rendre monnayable le service auquel sont astreints les absents. Pour ce faire, "les villages d'origine envoient des listes avec les noms des membres de chaque village, afin de collecter l'argent des fêtes ${ }^{228)}$.' D'autres communautés permettent aux migrants de payer et de déléguer à un membre de leur famille (parents, frères, beaux-parents), voire à des personnes étrangères à la famille, l'exercice de la fonction au sein de la communautéter). 
La migration a assoupli le modèle en y incorporant des membres de la communauté qui autrefois n'avaient pas le droit d'y participer : les vieillards et les femmes. Ces dernières se sont vues dans l'obligation d'assumer des fonctions qui incomberaient à leurs maris et à leurs fils si ceux-ci étaient présents ${ }^{(30)}$. Si certains auteurs ont suggéré que la visibilité des femmes dans le système de distribution des responsabilités au sein de la communauté pourrait traduire un pouvoir accru des femmes, force est de reconnaître qu'il s'agit d'une simple suppléance destinée à éviter les sanctions qu'entraînerait pour le groupe domestique le fait de ne pas remplir ses obligations, sans que la représentante obtienne un quelconque pouvoir en contrepartie ${ }^{(31)}$. Il s'agit, affirme Velázquez, d'un "type de transfert temporaire de la citoyenneté de l'homme migrant à la femme $e^{(32) " . ~ M a l g r e ́ ~ d e ~ t e l l e s ~ a d a p-~}$ tations, le système de distribution des responsabilités au sein de la communauté présente des fissures, et on voit de plus en

\section{Les communautés} - notamment celles des États de Puebla et Oaxaca, où les us et coutumes sont demeurés bien vivants - ont décidé d’infliger tout un ensemble de sanctions aux migrants qui transgressent les règles, ainsi qu'à leurs parents restés au pays. plus de migrants se refuser à revenir ou à payer leur quote-part. L'absence prolongée qui, inéluctablement, finit par affaiblir les liens sociaux, ainsi que l'établissement indéfini des familles dans les lieux d'accueil, ont engendré des situations qui ne favorisent nullement le financement des fêtes.

Dans ces conditions, les communautés - notamment celles des États de Puebla et Oaxaca, où les us et coutumes sont demeurés bien vivants - ont décidé d'infliger tout un ensemble de sanctions aux migrants qui transgressent les règles, ainsi qu'à leurs parents restés au pays; parmi celles-ci figurent, évidemment, les amendes à verser en liquide $^{(33)}$; s'ils ne les paient pas, affirment les autorités, "ils ne pourront plus revenir au pays après toutes ces années d'absence ${ }^{(34) " . ~ D ' a u t r e s ~ s a n c t i o n s ~ c o n s i s t e n t ~ a ̀ ~ l e u r ~ r e f u s e r ~}$ certains biens et services, et à les déchoir de leurs droits communautaires. Ceux qui rechignent à s'acquitter de leurs responsabilités se voient refuser la délivrance de certains documents (actes de naissance ou de décès, certificats scolaires) dont ils ont besoin dans les lieux d'accueil ; on leur coupe les services de distribution de l'énergie électrique et de l'eau ; on les menace de les priver de leurs droits agraires. Certains propriétaires ont été expropriés sous prétexte que les maisons étaient construites sur des ejidos $^{(35)}$. On leur fait savoir qu'en cas de décès leurs restes ne pourront pas être rapatriés au village, ni être enterrés au cimetière municipal ${ }^{(36)}$. Un migrant d'Oaxaca m'a fait part de ce qu'il considérait comme l'une des pires représailles qui pouvait être prise à l'encontre de ceux qui ne revenaient pas au village et ne payaient pas leur quote- 
part : lorsqu'un membre de leur famille mourait dans le village, il n'y avait aucune fanfare, ni personne pour l'accompagner jusqu'à sa dernière demeure. Cruelle offense dans ces communautés où, comme dans la sienne, la mort fait l'objet d'un très important rituel communautaire. En conséquence de quoi on avait commencé à devoir louer des camionnettes pour aller enterrer les morts, ce qui ne s'était jamais vu auparavant : autant de mesures désespérées de la part des communautés, qui ont toujours moins de ressources et d'emprise sur leurs membres, afin de maintenir les systèmes traditionnels de pouvoir au moyen de contraintes et d'exigences excessives. De nombreux auteurs ont mis l'accent sur les aspects positifs du capital social, permettant d'encourager et de faciliter la migration. Toutefois, Alejandro Portes a souligné les aspects négatifs que peut présenter le capital social ${ }^{(37)}$; parmi ceux-ci, il mentionne les exigences et contraintes excessives dont les migrants et leurs familles peuvent faire l'objet de part et d'autre de la frontière, grâce à la densité et à la solidité des réseaux sociaux. Cette caractéristique du capital social peut contribuer à expliquer pourquoi, à un moment donné, les exigences peuvent atteindre un niveau tel que les individus concernés décident de changer les règles du jeu, voire de les méconnaître.

\section{Les nouvelles règles des migrants}

Les changements s'expriment de trois manières différentes. La première est le mécontentement des migrants, qui a donné lieu à des situations et des affrontements jamais vus auparavant. Rivermar Pérez ${ }^{(38)}$ signale le cas d'un migrant de Xoyatla (État de Puebla) qui "s'est refusé à céder aux menaces des autorités" et a eu recours à la Commission des droits de l'homme afin de dénoncer ce qu'il estimait être une violation de ses droits fondamentaux. La Commission s'est prononcée en sa faveur, obligeant la communauté à modérer ses exigences envers les migrants. Dans d'autres cas, les migrants ont refusé de payer leur quote-part et rompu tout lien avec la communauté. Au Michoacán, par exemple, il y a certains migrants qui ne peuvent plus retourner dans leurs villages, ce qui les oblige à se réunir autre part avec leur famille. Sánchez a signalé que lors d'une consultation effectuée auprès des membres d'une communauté zapotèque d'Oaxaca, la plupart des migrants se sont montrés favorables à une modification du système des us et coutumes, ainsi qu'à une réduction du nombre des fonctions à remplir au sein de la communautée ${ }^{(39)}$.

En second lieu, le changement s'exprime à travers les femmes qui restent au pays. Obligées d'assumer les fonctions masculines, elles ont vu s'alourdir leurs responsabilités, sans que cela n'entraîne pour elles un surplus de pouvoir, ni une rétribution quelconque. Ceci les a conduites à négliger l'éducation de leurs enfants, 
ainsi que certaines activités lucratives auxquelles elles se livrent, état de choses avec lequel elles ne sont pas d'accord ${ }^{(40)}$.

En troisième lieu, on voit les nouvelles générations - les enfants et les jeunes nés ou ayant grandi au sein de la diaspora - se détourner des obligations et des coûts qu'implique le système de la distribution des responsabilités communautaires, étant donné le sacrifice économique supplémentaire qu'il représente pour les familles dans les lieux d'accueil. Les hommes qui rentraient au pays afin de s'acquitter de leurs responsabilités civiques à San Jerónimo Progreso, une communauté mixtèque, reconnaissaient cet état de fait: "Ma femme ou mes filles, ma mère et ma belle-mère travaillent pour m'aider ${ }^{(41)}$ ". Les jeunes filles de cette communauté établies à Tijuana étaient en désaccord avec cette règle. C'est ainsi que Rosalía, une étudiante dont le père était apparemment en train de remplir ses obligations communautaires à San Jerónimo Progreso, confia ce qui suit à Ruiz Robles : "Ce que le village exige de nous, ou ce que les autorités nous demandent de faire, ça ne me semble pas juste...jai demandé à mon père s'il n'y avait pas moyen de changer tout cela, parce qu'enfin ce n'est pas une vie, ça, que mon père soit obligé d'être là-bas, tandis qu'ici ma mère, ou bien les enfants... en train de trimer... et les gens non plus ne font rien pour étudier, ils n'ont aucune ambition dans la vie, parce qu'ils se disent : nous, on travaille pour que dans deux ou trois ans papa soit réélu et doive à nouveau remplir ses obligations dans la communauté... ; autrement dit, on doit travailler au cas où il arriverait quelque chose comme ça ${ }^{(42)}$..." Les sanctions infligées à ceux qui ne s'acquittaient pas de leurs responsabilités étaient, en effet, terribles : "On leur enlève leurs terres ou leurs maisons ${ }^{(43)}$." Les membres des nouvelles générations sont plus réticents à payer ou à s'endetter afin que leurs parents continuent à participer aux fêtes patronales comme le veut la tradition, surtout s'ils ne rentreront pas au pays. En 2005, les dépenses qu'impliquait une mayordomía dans une communauté rurale de l'État de Puebla, s'élevaient à quinze mille dollars : cette somme, destinée à payer les danseurs, les musiciens, les feux d'artifice, les $\operatorname{castillos}^{(44)}$, à donner à manger à tout le village pendant deux jours, représentait les économies de toute une année de travail ${ }^{(45)}$. La situation est difficile pour tout le monde, y compris, bien entendu, pour l'avenir des fêtes patronales. Si, dans un premier temps, les migrants ont été surpris par les sanctions infligées par les communautés d'origine, ils ont fini par mettre eux aussi leur atout sur la table: si on les prive de leurs droits dans les villages, ils refuseront toute aide aux gens de leur communauté lorsqu'ils arriveront dans le pays d'accueil et auront besoin de quelqu'un qui les héberge et les aide à trouver du travail. Mais c'est là une décision hautement risquée. En effet, les migrants sans papiers se font beaucoup de souci pour leurs propriétés, surtout pour leurs maisons, qui représentent une sécurité face à l'éventualité d'être déportés vers le Mexique - une éventualité qui n'a rien d'invraisemblable par les temps qui courent. 


\section{Conclusion}

La crise généralisée du monde rural a obligé les communautés à chercher de nouveaux modes d'insertion économique et sociale leur permettant non seulement de survivre, ne serait-ce que de façon précaire, mais aussi de conserver les liens avec une diaspora dont l'absence est de plus en plus prolongée. L'idéologie ou culture du retour s'affronte et s'oppose à des pratiques qui traduisent des permanences indéfinies, une tendance de plus en plus marquée à s'établir dans les lieux d'accueil, ce qui a pour résultat de modifier les scénarios, les sens et les acteurs traditionnels des fêtes patronales.

La fête patronale, qui dota jadis les villages d'un temps unique et distinctif, continue à être reconnue par les migrants et leurs descendants comme une occasion de retour, ne serait-ce que temporaire. Mais la fête patronale redéfinit également les rapports entre ceux qui sont restés et ceux dont se compose la diaspora, avec ses différentes générations, en particulier de nombreux jeunes qui connaissent ou non les croyances, les sens, les valeurs traditionnelles qui se cristallisaient dans la fête patronale.

La continuité et les changements des fêtes patronales apparaissent liés à deux projets divergents. D'une part, le projet qui consiste à promouvoir la fête comme espace de distraction, de spectacle et de liberté pour les jeunes - une liberté que l'on ne trouve pas dans les villes, à plus forte raison aux États-Unis, une liberté qui n'a pas grandchose à voir avec le sens religieux et rituel, ni avec les traditions que d'autres aimeraient conserver ${ }^{(46)}$.Ce projet s'avère très conflictuel dans la région historique de la migration, où l'Église catholique conserve un grand poids institutionnel, normatif et moral. La promotion d'une fête patronale transformée en spectacle et en espace de liberté pour les jeunes oppose les autorités ecclésiastiques, les habitants des villages et les organisations catholiques aux autorités - locales et régionales - qui s'efforcent de mettre en valeur les attraits touristiques et récréatifs dont peuvent être investis les espaces ruraux pour les descendants des migrants, pour les habitants des villes et pour tous ceux qui viennent de l'extérieur.

D'autre part, le projet des communautés rurales et des autorités locales, qui mettent en ceuvre tout un ensemble de contraintes afin de maintenir en vie les fêtes, le système de distribution des responsabilités au sein de la communauté, dans le but de récupérer leurs migrants, comme c'est le cas des communautés à forte tradition indigène des nouvelles régions migratoires. Ce projet crée un antagonisme frontal entre, d'une part, les habitants des communautés et les mécanismes traditionnels du pouvoir local et, d'autre part, les migrants, ce qui pousse ces derniers à prendre des décisions radicales concernant leurs rapports avec leurs communautés d'origine.

Il ne fait aucun doute que la fête patronale est en pleine transformation. Son avenir dépendra des solutions, certainement multiples et diverses, qui seront adoptées et mises 
en ceuvre par les communautés et leurs diasporas afin de gérer la migration, ainsi que l'absence sinon définitive, tout au moins prolongée de leur population.

\section{Traduit de l'espagnol par Jean Hennequin}

\section{Notes}

1. María Eugenia D'Aubeterre, "Aquí respetamos a nuestros esposos". Migración masculina y trabajo femenino en una comunidad de origen nahua del Estado de Puebla", in Marina Ariza et Alejandro Portes (dir.) El país transnacional. Migración mexicana y cambio social a través de la frontera. México, Unam, 2007, pp. 513-544 ; Soledad González Montes, "La fiesta interminable: celebraciones públicas y privadas en un pueblo campesino del Estado de México", in Pilar Gonzalvo Aizpuru (dir.), Historia de la vida cotidiana en México, t. V, vol. 1, n XX, México, El Colegio de México, FCE, 2006,pp. 365-397 ; Shinji Hirai, Economía política de la nostalgia. Un estudio sobre la transformación del paisaje en la migración transnacional entre México y Estados Unidos, México, UAM-I, 2009.

2. José María Arguedas, Las comunidades de España y del Perú, Lima, Universidad Nacional Mayor de San Marcos, 1968 , p. 221.

3. María Leticia Rivermar Pérez, Etnicidad y migración internacional. El caso de una comunidad nahua en el Estado de Puebla, Puebla, Benemérita Universidad Autónoma de Puebla, 2008.

4. La mayordomía est la fonction exercée par le mayordomo, notable local chargé de patronner les fêtes de la communauté et responsable en tant que tel de la préparation, de l'organisation et du déroulement de celles-ci pendant une période déterminée, au terme de laquelle il transmet cette responsabilité à son successeur [N. du T.].

5. Frank Cancian, Economía y prestigio en una comunidad maya. El sistema de cargos en Zinacantán, México, INI, 1976 ; María Ana Portal Ariosa, Ciudadanos desde el pueblo. Identidad urbana y religiosidad popular en San Andrés Totoltepec, Tlalpan, México, D.F., México, Conaculta, UAM-Ixtapalapa, 1997.

6. Cristina Oehmichen, "Parentesco y matrimonio en la comunidad extendida: el caso de los mazahuas", in Alteridades, vol. 12, n 24, México, UAM-I, 2002, pp. 61-74 ; Joaquín Peña Piña, "Migración, remesas y estrategias de reproducción. Mujeres esposas de migrantes y relaciones de género en la región indígena mam del Soconusco, Chiapas", in Blanca Suárez et Emma Zapata Martelo (dir.), Remesas. Milagros y mucho más realizan las mujeres indígenas y campesinas, vol. II, México, Gimtrap, 2004, pp. 33-76; Laura Velasco Ortiz, El regreso de la comunidad: migración indígena y agentes étnicos: los mixtecos en la frontera México-Estados Unidos, México, El Colegio de México, 2002.

7. Patricia Arias, Del arraigo a la diáspora. Dilemas de la familia rural, México, Miguel Angel Porrúa, 2009 ; Jorge Durand et Douglas S. Massey, Clandestinos. Migración México-Estados Unidos en los albores del siglo XXI, México, Universidad Autónoma de Zacatecas-Miguel Angel Porrúa, 2003.

8. María Eugenia D'Aubeterre, "Tiempos de espera: emigración masculina, ciclo doméstico y situación de las mujeres en San Miguel Acuexcomac, Puebla", in Soledad González Montes et Vania Salles (dir.), Relaciones de género y transformaciones agrarias, México, El Colegio de México, 1995, pp. 255-297 ; Jorge Durand et Douglas S. Massey, Clandestinos. Migración México-Estados Unidos en los albores del siglo XXI, op. cit.; Rosío Córdova Plaza, Cristina Núñez Madrazo et David Skerritt Gardner, Migración internacional, crisis agrícola y transformaciones culturales en la región central de Veracruz, México, Plaza y Valdés, 2008 ; Cristina Oehmichen Bazán, Identidad, género y relaciones interétnicas. Mazahuas en la ciudad de México, México, UNAM, 2005 ; Laura Velasco Ortiz, El regreso de la comunidad: migración indígena y agentes étnicos: los mixtecos en la frontera México-Estados Unidos, op. cit.

9. Ejidos : terres communales attribuées aux villages mexicains dans le cadre de la Réforme agraire des années 19201940 [N. du T.].

10. Patricia Arias, Del arraigo a la diáspora. Dilemas de la familia rural, op. cit.

11. IRCA : Immigration Reform and Control Act [N. du T.].

12. Jorge Durand et Douglas S. Massey, Clandestinos. Migración México-Estados Unidos en los albores del siglo XXI, op. cit.

13. Migradollar: se réfère aux dollars envoyés par les travailleurs émigrés à leurs communautés d'origine [N. du T.]. 
14. María Eugenia D’Aubeterre, "Tiempos de espera: emigración masculina, ciclo doméstico y situación de las mujeres en San Miguel Acuexcomac, Puebla", op. cit.

15. Julia Pauli, "Que vivan mejor aparte": migración, estructura familiar y género en una comunidad del México central", in David Robichaux (dir.), Familias mexicanas en transición, México, Universidad Iberoamericana, 2007, pp. $87-116$.

16. Martha Judith Sánchez, "La importancia del sistema de cargos en el entendimiento de los flujos migratorios indígenas", in Marina Ariza et Alejandro Portes (dir.), El país transnacional. Migración mexicana y cambio social a través de la frontera, México, UNAM, 2007, pp. 349-390.

17. Patricia Arias, Del arraigo a la diáspora. Dilemas de la familia rural, op. cit.; María da Gloria Marroni, "Los cambios en la sociedad rural y el trabajo doméstico en Atlixco, Puebla, 1940-1990", in Heather Fowler-Salamini et Mary Kay Vaughan (dir.), Mujeres del campo mexicano. 1850-1990, Zamora, El Colegio de Michoacán, 2003, pp. 323-342.

18. Jorge Durand et Douglas S. Massey, Clandestinos. Migración México-Estados Unidos en los albores del siglo XXI, op. cit.

19. Douglas S. Massey, Jorge Durand et Fernando Riosmena, "Capital social, política social y migración desde comunidades tradicionales y nuevas comunidades de origen en México", in Revista Española de Investigaciones Sociológicas, $\mathrm{n}^{\circ}$ 116, 2006, pp. 97-121.

20. Jorge Durand, Más allá de la línea. Patrones migratorios entre México y Estados Unidos, México, Conaculta, 1994, p. 317 .

21. Shinji Hirai, Economía política de la nostalgia. Un estudio sobre la transformación del paisaje en la migración transnacional entre México y Estados Unidos, op. cit.

22. Morán Quiroz, Luis Rodolfo, "Devociones populares y comunidades transnacionales", in Olga Odgers Ortiz et Juan Carlos Ruiz Guadalajara (dir.), Migración y creencias. Pensar las religiones en tiempo de movilidad, México, El Colegio de la Frontera Norte, El Colegio de San Luis, Miguel Angel Porrúa, 2009, pp. 75-96.

23. Elizabeth Juárez Cerdí, "Viviendo en un espacio ajeno. La Semana Santa entre migrantes mexicanos en Immokalee, Florida”, in Relaciones, vol. XXX, n 119, été 2009, pp. 135-158.

24. Liliana Rivera Sánchez, "Cuando los santos también migran. Conflictos transnacionales por el espacio y la pertenencia", in Migraciones Internacionales, vol. 3, n 4, juillet-décembre 2006, pp. 35-59.

25. María Eugenia D’Aubeterre, "Aquí respetamos a nuestros esposos". Migración masculina y trabajo femenino en una comunidad de origen nahua del Estado de Puebla", op. cit.

26. María Leticia Rivermar Pérez, Etnicidad y migración internacional. El caso de una comunidad nahua en el Estado de Puebla, op. cit.; María Cristina Velázquez, "Eslabones entre el migrante y su pueblo: las mujeres en la nueva institucionalidad comunitaria indígena de la Mixteca oaxaqueña”, in Blanca Suárez et Emma Zapata Martelo (dir.), Ilusiones, sacrificios y resultados. El escenario real de las remesas de emigrantes a Estados Unidos, México, GIMTRAP, 2007, pp. 259-300.'

27. Martha Judith Sánchez, "La importancia del sistema de cargos en el entendimiento de los flujos migratorios indígenas", op. cit.

28. Laura Velasco Ortiz, El regreso de la comunidad: migración indígena y agentes étnicos: los mixtecos en la frontera México-Estados Unidos, op. cit., p. 129 ; Martha Judith Sánchez, "La importancia del sistema de cargos en el entendimiento de los flujos migratorios indígenas", op. cit.

29. Liliana Rivera Sánchez, "Cuando los santos también migran. Conflictos transnacionales por el espacio y la pertenencia", op. cit.; Martha Judith Sánchez, "La importancia del sistema de cargos en el entendimiento de los flujos migratorios indígenas", op. cit.; María Cristina Velázquez, "Eslabones entre el migrante y su pueblo: las mujeres en la nueva institucionalidad comunitaria indígena de la Mixteca oaxaqueña", op. cit.

30. Beatriz Canabal Cristiani, "Y entonces, yo me quedé a cargo de todo...". La mujer rural hoy", in Beatriz Canabal Cristiani, Gabriela Contreras Pérez et Arturo León López (dir.), Diversidad rural. Estrategias económicas y procesos culturales, México, UAM-X-Plaza y Valdés Editores, 2006, pp. 19-37 ; Martha Judith Sánchez, "La importancia del sistema de cargos en el entendimiento de los flujos migratorios indígenas", op. cit.

31. Patricia Arias, Del arraigo a la diáspora. Dilemas de la familia rural, op. cit.; María Eugenia D'Aubeterre, “'Aquí respetamos a nuestros esposos'. Migración masculina y trabajo femenino en una comunidad de origen nahua del Estado de Puebla", op. cit.

32. María Cristina Velázquez, "Eslabones entre el migrante y su pueblo: las mujeres en la nueva institucionalidad comunitaria indígena de la Mixteca oaxaqueña", op. cit., p. 275. 
33. María Leticia Rivermar Pérez, Etnicidad y migración internacional. El caso de una comunidad nahua en el Estado de Puebla, op. cit.

34. Miriam Castaldo Cossa, "En torno al concepto de migración y remesas: presencia, ausencia y apariencia", in Blanca Suárez et Emma Zapata Martelo (dir.), Remesas. Milagros y mucho más realizan las mujeres indígenas y campesinas, vol. II., México, Gimtrap, 2004, p. 241.

35. María Leticia Rivermar Pérez, Etnicidad y migración internacional. El caso de una comunidad nahua en el Estado de Puebla, op. cit.

36. Cristina Oehmichen, "Parentesco y matrimonio en la comunidad extendida: el caso de los mazahuas", op. cit.; María Leticia Rivermar Pérez, Etnicidad y migración internacional. El caso de una comunidad nahua en el Estado de Puebla, op. cit.

37. Marina Ariza et Alejandro Portes (dir.), El país transnacional. Migración mexicana y cambio social a través de la frontera, México, UNAM, 2007.

38. María Leticia Rivermar Pérez, Etnicidad y migración internacional. El caso de una comunidad nahua en el Estado de Puebla, op. cit.

39. Martha Judith Sánchez, "La importancia del sistema de cargos en el entendimiento de los flujos migratorios indígenas", op. cit.

40. María Eugenia D'Aubeterre, "Aquí respetamos a nuestros esposos". Migración masculina y trabajo femenino en una comunidad de origen nahua del Estado de Puebla", op. cit.; María Cristina Velázquez, "Eslabones entre el migrante y su pueblo: las mujeres en la nueva institucionalidad comunitaria indígena de la Mixteca oaxaqueña", op. cit.

41. Ruiz Robles, Raúl René, "San Jerónimo Progreso: migración y remesas. Un sistema político sustentado por ellas", in Blanca Suárez y Emma Zapata Martelo (dir.), Remesas. Milagros y mucho más realizan las mujeres indígenas y campesinas, vol. II, México, Gimtrap, 2004, p. 20.

42. Ibid., p. 20.

43. Ibid., p. 21.

44. Castillo (littéralement "château"): structure en bois de plusieurs mètres de hauteur, comportant toute une série de feux d'artifice et de pétards qui sont tirés les uns après les autres [N. du T.].

45. Liliana Rivera Sánchez, "Cuando los santos también migran. Conflictos transnacionales por el espacio y la pertenencia", op. cit.

46. Shinji Hirai, Economía política de la nostalgia. Un estudio sobre la transformación del paisaje en la migración transnacional entre México y Estados Unidos, op. cit. 